\title{
SCIENTIFIC REPORTS

\section{OPEN Application of Japanese guidelines for gestational weight gain to multiple pregnancy outcomes and its optimal range in 101,336 Japanese women}

\begin{abstract}
Kyoko Nomura $\mathbb{1}^{1 *}$, Kengo Nagashima ${ }^{2}{ }^{2}$, Shunji Suzuki ${ }^{3}$ \& Hiroaki Itoh ${ }^{4}$
This study was performed to investigate whether the Japanese guidelines for gestational weight gain (GWG) can be used to determine the risks of multiple pregnancy outcomes and estimate optimal GWG in 101,336 women with singleton pregnancies in 2013. Multivariable logistic regression analyses indicated that the risks associated with low birth weight, small for gestational age, and preterm birth increased significantly with weight gain below the Japanese guidelines, and the risks of macrosomia and large for gestational age increased with weight gain above the guidelines regardless of Asian-specific prepregnancy body mass index (BMI). The GWG cutoff points estimated from the adjusted area under the receiver operating characteristics curve $>0.6$ corresponded to $10-13.8 \mathrm{~kg}$ in underweight women with pre-pregnancy $B M I<18.5 \mathrm{~kg} / \mathrm{m}^{2} ; 10-13.7 \mathrm{~kg}$ in normal weight women with pre-pregnancy BMI $18.5-22.9 \mathrm{~kg} / \mathrm{m}^{2} ; 8.5-11.4 \mathrm{~kg}$ in overweight women with pre-pregnancy BMI $23-24.9 \mathrm{~kg} / \mathrm{m}^{2}, 5-13.3 \mathrm{~kg}$ in obese women with pre-pregnancy BMI $25-29.9 \mathrm{~kg} / \mathrm{m}^{2}$; and $4.7 \mathrm{~kg}$ in obese women with pre-pregnancy $\mathrm{BMI} \geq 30 \mathrm{~kg} / \mathrm{m}^{2}$. The optimal GWG ranges proposed by the present study are slightly higher than those recommended by the current Japanese guidelines.
\end{abstract}

In Japan, one quarter of women of reproductive age are underweight, as defined by body mass index $(\mathrm{BMI})<18.5 \mathrm{~kg} / \mathrm{m}^{2}$, and underweight women are at risk of delivering low-birth-weight (LBW) infants ${ }^{1,2}$. Recent studies have implied that epigenetic alterations are induced by the uterine environment of underweight mothers. The "developmental origins of health and disease" hypothesis ${ }^{3}$ proposes that fetal undernutrition has permanent effects on growth and can cause lifestyle-related diseases such as type 2 diabetes and cardiovascular disease ${ }^{4}$.

In 2018, the incidence of LBW infants was higher in Japan (9.4\%) than the average among Organization for Economic Cooperation and Development (OECD) countries $(6.5 \%)^{5}$. This high incidence of small babies has been observed for over two decades in $\mathrm{Japan}^{6}$, and pre-pregnancy underweight mothers and poor weight gain during pregnancy are major related factors ${ }^{7}$. The Japanese government has taken measures to reduce the number of LBW infants since 2001 in a national campaign to promote maternal and child health in the $21^{\text {st }}$ century, but the incidence increased between 2001 and 2014 and is still far above the average of OECD countries ${ }^{8}$.

The Japanese Ministry for Health, Labor, and Welfare introduced gestational weight gain (GWG) recommendations in 2001 for appropriate weight gain during pregnancy ${ }^{9}$ 9-12 kg for underweight and 7-12 kg for normal-weight women, and $\leq 5 \mathrm{~kg}$ (depending on individual cases) for overweight and obese women. A recent study ${ }^{10}$ that investigated attitudes to strict adherence to the GWG guidelines reported that $80 \%$ of pregnant women considered avoiding excessive GWG important "for ease of delivery and/or her health." However, those who limited GWG to below or within the guidelines were more likely to have LBW infants than were those whose GWG exceeded the guidelines. A study ${ }^{2}$ of 97,157 pregnant Japanese women using the Japanese Society of

${ }^{1}$ Department of Environmental Health Science and Public Health, Akita University Graduate School of Medicine, Akita City, 010-8543, Japan. ${ }^{2}$ Research Center for Medical and Health Data Science, The Institute of Statistical Mathematics, Tachikawa City, 190-0862, Japan. ${ }^{3}$ Department of Obstetrics and Gynecology, Japanese Red Cross Katsushika Maternity Hospital, Katsushika Ku, 124-0012, Japan. ${ }^{4}$ Department of Obstetrics and Gynecology, Hamamatsu University School of Medicine, Hamamatsu, 431-3192, Japan. *email: knomura@med.akita-u.ac.jp 
Obstetrics and Gynecology (JSOG) Successive Pregnancy Birth Registry System showed that the average weight gain during pregnancy was $10.3 \mathrm{~kg}$ in underweight and $10.1 \mathrm{~kg}$ in normal-weight mothers, implying that both obstetricians and pregnant women in Japan follow the government guidelines.

Several studies have investigated the combination of pre-pregnancy BMI and total GWG associated with pregnancy outcomes with various assessments, including statistical interactions ${ }^{11}$, stratification by pre-pregnancy $\mathrm{BMI}_{\text {level }}{ }^{2,12,13}$, and the speed of weight gain ${ }^{14-17}$. Furthermore, some recent studies investigated the applicability of the Institute of Medicine (IOM) ${ }^{18}$ guidelines to Asian women ${ }^{2,17}$. However, the guidelines ${ }^{18}$ were developed for safe pregnancy outcomes in Western countries, where the prevalence of obesity is higher. Excessive weight gain in small and lean Asian women may result in additional adverse events, such as excessive hemorrhage and pregnancy-induced hypertension ${ }^{19}$, and therefore the applicability of the IOM guidelines to Asian women still requires scientific justification ${ }^{20}$.

We previously investigated whether the IOM and Japanese guidelines could identify the risks of small for gestational age (SGA) and large for gestational age (LGA) births associated with GWG below and above the recommended level and estimated the optimal GWG in a population of 8,152 Japanese women recruited at a single hospital $^{21}$. The present study was performed to investigate whether the current Japanese GWG recommendations can significantly determine the risks of multiple pregnancy outcomes using the World Health Organization (WHO) Asian-specific pre-pregnancy BMI classification ${ }^{22}$, and to estimate the optimal weight gain range using a GWG cutoff point associated with the risk of multiple pregnancy outcomes by multivariate adjusted receiver operating characteristic (ROC) curve analysis ${ }^{23}$. We focused specifically on the Japanese guidelines, as the incidence of LBW infants in Japan has been high among OECD countries for over two decades, and scientific evidence is urgently required to determine whether the current guidelines should be changed.

\section{Results}

Table 1 shows the basic characteristics of the study population according to the WHO Asian-specific pre-pregnancy BMI classification. Among 101,336 subjects included in the analyses, 18.1\% were underweight (pre-pregnancy BMI $<18.5 \mathrm{~kg} / \mathrm{m}^{2}$, mean age 31.0 years), $9.9 \%$ were overweight (pre-pregnancy BMI $23-24.9 \mathrm{~kg} / \mathrm{m}^{2}$, mean age 31.9 years), $7.8 \%$ were obese I (pre-pregnancy BMI $25-29.9 \mathrm{~kg} / \mathrm{m}^{2}$, mean age 32.6 years), and $3.1 \%$ were obese II (pre-pregnancy BMI $\geq 30 \mathrm{~kg} / \mathrm{m}^{2}$, mean age 32.3 years). The mean (SD) GWG was $10.2(3.7) \mathrm{kg}$ among underweight women, $10.2(3.9) \mathrm{kg}$ among normal-weight women, 9.5 (4.5) kg among overweight women, 7.9 (5) $\mathrm{kg}$ among obese I women, and $5.2(5.7) \mathrm{kg}$ among obese II women. These values for underweight and normal-weight women fell into the range recommended by the Japanese Ministry of Health, Labour, and Welfare, but those for overweight and obese women exceeded the recommended ranges. Underweight women tended to have SGA and LBW infants, whereas overweight and obese women tended to have LGA infants, macrosomia, gestational diabetes mellitus (GDM), pregnancy-induced hypertension, and postpartum hemorrhage with cesarean section (PPH-CS).

Table 2 shows the risks of various pregnancy outcomes associated with GWG below and above the government recommendations comparing with weight gain within the recommendations among underweight and normal-weight women. Compared to women who gained weight within the government recommended range, women with weight gain below the recommended ranges had a significantly increased risk of having small babies (i.e., SGA and LBW) and preterm birth (all adjusted $p<0.0001$ ). Women with weight gain above the recommendations had significantly increased risks of LGA, macrosomia, pregnancy-induced hypertension, $\mathrm{PPH}$ with vaginal delivery (PPH-VD), and PPH-CS (all adjusted $p<0.0001$ ).

Table 3 shows the risks of various pregnancy outcomes associated with weight gain below or above $5 \mathrm{~kg}$ in overweight and obese women. The risks of SGA, LBW, and preterm birth were significantly increased with $\leq 5 \mathrm{~kg}$ weight gain, whereas the risks of LGA, macrosomia (all adjusted $p<0.0001$, except for overweight women), pregnancy-induced hypertension, and PPH-CS (all adjusted $p<0.0001$, except for obese II women) increased significantly with GWG $>5 \mathrm{~kg}$.

The risk of GDM decreased significantly with weight gain $>12 \mathrm{~kg}$ in normal-weight women [OR $0.72,95 \%$ confidence interval (CI): $0.64-0.81$, and with weight gain $>5 \mathrm{~kg}$ in overweight women (OR $0.49,95 \% \mathrm{CI}$ : $0.41-$ 0.59 ), obese I women (OR 0.57, 95\% CI: 0.50-0.65) and obese II women (OR 0.69, 95\% CI: 0.58-0.81).

Table 4 shows the GWG cutoff points based on the area under the curve (AUC) according to various pregnancy outcomes. After adjusting for covariates selected in stepwise multivariable logistic regression analyses, pregnancy outcomes with the lower boundary of a 95\% CI of AUC $>0.6$ included SGA, preterm birth, LGA, and macrosomia in underweight women; preterm birth, LGA, and macrosomia in normal-weight women; preterm birth, LGA, and macrosomia in overweight women; preterm birth, LGA, and macrosomia in obese I women; and macrosomia in obese II women. Based on the largest Youden's index calculated by bootstrapped sensitivity and specificity, we estimated the adjusted GWG cutoff points to be $10 \mathrm{~kg}$ for SGA and preterm birth, $12 \mathrm{~kg}$ for LGA, and $13.8 \mathrm{~kg}$ for macrosomia, (i.e., range $10-13.8 \mathrm{~kg}$ ) in underweight women; to be $10 \mathrm{~kg}$ for preterm birth, $11.7 \mathrm{~kg}$ for LGA, and $13.7 \mathrm{~kg}$ for macrosomia, (i.e., range $10-13.7 \mathrm{~kg}$ in normal-weight women; to be $8.5 \mathrm{~kg}$ for preterm birth, $11.3 \mathrm{~kg}$ for LGA, and $11.4 \mathrm{~kg}$ for macrosomia, (i.e., range $8.5-11.4 \mathrm{~kg}$ ) in overweight women; to be $5 \mathrm{~kg}$ for preterm birth, $8 \mathrm{~kg}$ for LGA, and $13.3 \mathrm{~kg}$ for macrosomia, (i.e., range 5-13.3 kg) in obese I women; and to be $4.7 \mathrm{~kg}$ for macrosomia in obese II women.

Table 5 shows the results of sensitivity analyses when women with preterm birth were excluded. The cutoff points for GWG with a lower boundary of $95 \%$ CI of the adjusted AUC $>0.6$ corresponded to $11.2 \mathrm{~kg}$ for SGA, $10 \mathrm{~kg}$ for LBW, $12.6 \mathrm{~kg}$ for LGA, and $12 \mathrm{~kg}$ for macrosomia, (i.e., range 10-12.6 kg) in underweight women; to $10.6 \mathrm{~kg}$ for SGA, $10.4 \mathrm{~kg}$ for LBW, $10 \mathrm{~kg}$ for LGA, and $12.7 \mathrm{~kg}$ for macrosomia, (i.e., range $10-12.7 \mathrm{~kg}$ ) in normal-weight women; to $11.3 \mathrm{~kg}$ for LGA, $10 \mathrm{~kg}$ for macrosomia, (i.e., range $11.3-11.4 \mathrm{~kg}$ ) in overweight women, $11.5 \mathrm{~kg}$ for LGA, and $14.1 \mathrm{~kg}$ for macrosomia, (i.e., range $11.5-14.1 \mathrm{~kg}$ ) in obese I women; and to $5.8 \mathrm{~kg}$ for macrosomia in obese II women. 


\begin{tabular}{|c|c|c|c|c|c|c|c|c|c|c|}
\hline & \multicolumn{10}{|c|}{ Asian specific pre-pregnancy BMI } \\
\hline & \multicolumn{2}{|c|}{$<18.5 \mathrm{~kg} / \mathrm{m}^{2}$} & \multicolumn{2}{|c|}{$18.5-22.9 \mathrm{~kg} / \mathrm{m}^{2}$} & \multicolumn{2}{|c|}{$23.0-24.9 \mathrm{~kg} / \mathrm{m}^{2}$} & \multicolumn{2}{|c|}{$25-29.9 \mathrm{~kg} / \mathrm{m}^{2}$} & \multicolumn{2}{|c|}{$30-\mathrm{kg} / \mathrm{m}^{2}$} \\
\hline & \multicolumn{2}{|c|}{ Underweight } & \multicolumn{2}{|c|}{ Normal weight } & \multicolumn{2}{|c|}{ Overweight } & \multicolumn{2}{|c|}{ Obese I } & \multicolumn{2}{|c|}{ Obese II } \\
\hline & \multicolumn{2}{|c|}{$n=18,382$} & \multicolumn{2}{|c|}{$n=61,960$} & \multicolumn{2}{|c|}{$\mathrm{n}=\mathbf{9 , 9 8 5}$} & \multicolumn{2}{|c|}{$n=7,873$} & \multicolumn{2}{|c|}{$n=3,136$} \\
\hline & $\begin{array}{l}\text { n or } \\
\text { mean }\end{array}$ & $\begin{array}{l}\text { (\%) or } \\
\text { SD }\end{array}$ & $\begin{array}{l}\text { n or } \\
\text { mean }\end{array}$ & \begin{tabular}{|l|}
$(\%)$ or \\
SD
\end{tabular} & $\begin{array}{l}\text { n or } \\
\text { mean }\end{array}$ & $\begin{array}{l}\text { (\%) or } \\
\text { SD }\end{array}$ & $\begin{array}{l}\text { n or } \\
\text { mean }\end{array}$ & \begin{tabular}{|l} 
(\%) or \\
SD
\end{tabular} & $\begin{array}{l}\text { n or } \\
\text { mean }\end{array}$ & \begin{tabular}{|l} 
(\%) or \\
SD
\end{tabular} \\
\hline Age, years & 31.0 & 5.4 & 31.9 & 5.3 & 32.4 & 5.5 & 32.6 & 5.5 & 32.3 & 5.3 \\
\hline Maternal height, $\mathrm{cm}$ & 158.6 & 5.4 & 158.2 & 5.4 & 157.7 & 5.5 & 157.8 & 5.7 & 158.1 & 5.8 \\
\hline Pre-pregnancy weight, $\mathrm{kg}$ & 44.3 & 3.6 & 51.2 & 4.5 & 59.4 & 4.3 & 67.3 & 5.9 & 84.3 & 10.1 \\
\hline Pre-pregnancy BMI, kg/m² & 17.6 & 0.8 & 20.4 & 1.2 & 23.9 & 0.6 & 27 & 1.4 & 33.7 & 3.4 \\
\hline Weight at delivery & 54.5 & 5.2 & 61.4 & 6.1 & 68.9 & 6.5 & 75.2 & 7.7 & 89.7 & 11.1 \\
\hline BMI at delivery & 21.7 & 1.7 & 24.5 & 1.9 & 27.7 & 1.9 & 30.2 & 2.3 & 35.9 & 3.7 \\
\hline Gestational week at delivery & 38.2 & 2.2 & 38.4 & 2.2 & 38.3 & 2.2 & 38.2 & 2.4 & 38.1 & 2.6 \\
\hline Gestational weight gain & 10.2 & 3.7 & 10.2 & 3.9 & 9.5 & 4.5 & 7.9 & 5 & 5.2 & 5.7 \\
\hline Nulliparity & 9,938 & $(54.2)$ & 31,669 & $(51.2)$ & 4,653 & $(46.8)$ & 3434 & $(43.7)$ & 1475 & $(47.1)$ \\
\hline \multicolumn{11}{|l|}{ Delivery Method } \\
\hline Vaginal Delivery & 14,089 & $(76.7)$ & 45,426 & $(73.3)$ & 6,685 & $(67.0)$ & 4,913 & $(62.4)$ & 1,758 & $(56.1)$ \\
\hline Caesarean Section & 4,155 & $(22.6)$ & 16,027 & $(25.9)$ & 3,221 & $(32.3)$ & 2,889 & $(36.7)$ & 1,341 & $(42.8)$ \\
\hline Other method & 138 & $(0.8)$ & 507 & $(0.8)$ & 79 & $(0.8)$ & 71 & $(0.9)$ & 37 & $(1.2)$ \\
\hline \multicolumn{11}{|l|}{ Smoking habits } \\
\hline Never & 16,800 & $(91.4)$ & 57,250 & $(92.4)$ & 9,070 & $(90.8)$ & 6,996 & $(88.9)$ & 2,660 & $(84.8)$ \\
\hline Past & 976 & $(5.3)$ & 3,087 & $(5.0)$ & 556 & (5.6) & 505 & $(6.4)$ & 267 & $(8.5)$ \\
\hline Current & 606 & $(3.3)$ & 1,623 & $(2.6)$ & 359 & (3.6) & 372 & $(4.7)$ & 209 & $(6.7)$ \\
\hline \multicolumn{11}{|l|}{ Pregnancy Outcome } \\
\hline SGA & 2,290 & $(12.5)$ & 5,121 & $(8.3)$ & 706 & $(7.1)$ & 553 & $(7.0)$ & 226 & $(7.2)$ \\
\hline Low birth weight & 3,635 & $(19.8)$ & 8,635 & $(13.9)$ & 1,272 & $(12.7)$ & 1091 & $(13.9)$ & 424 & (13.5) \\
\hline Preterm birth & 2,205 & $(12.0)$ & 6,199 & $(10.0)$ & 1,094 & $(11.0)$ & 915 & $(11.6)$ & 393 & (12.5) \\
\hline LGA & 885 & $(4.8)$ & 5,585 & $(9.0)$ & 1,282 & $(12.8)$ & 1,295 & $(16.5)$ & 688 & (21.9) \\
\hline Macrosomia & 45 & $(0.2)$ & 389 & $(0.6)$ & 102 & $(1.0)$ & 144 & $(1.8)$ & 102 & (3.3) \\
\hline GDM & 535 & $(2.9)$ & 2,322 & $(3.8)$ & 694 & $(7.0)$ & 1,036 & $(13.2)$ & 801 & $(25.5)$ \\
\hline $\begin{array}{l}\text { Pregnancy -induced } \\
\text { Hypertension }\end{array}$ & 613 & $(3.3)$ & 2,673 & $(4.3)$ & 674 & $(6.8)$ & 816 & (10.4) & 501 & (16.0) \\
\hline PPH with VD & 3644 & (19.8) & 13,156 & $(21.2)$ & 2,140 & (21.4) & 1,657 & $(21.1)$ & 694 & (22.1) \\
\hline PPH with CS & 893 & (4.9) & 4,605 & (7.4) & 972 & (9.7) & 910 & (11.6) & 437 & (13.9) \\
\hline
\end{tabular}

Table 1. Basic characteristics according to WHO Asian-specific BMI classification. BMI, Body Mass Index; SGA, small for gestational age; LGA, large for gestational age; GDM, Gestational Diabetes Mellitus; PPH with VD, postpartum hemorrhage with vaginal delivery; PPH with CS, postpartum hemorrhage with cesarean section.

\section{Discussion}

Utilizing the JSOG Successive Pregnancy Birth Registry, this study showed that the current Japanese GWG recommendations can significantly identify the risks of multiple pregnancy outcomes. Among these outcomes, the risks of SGA, LBW, and preterm birth significantly increased with insufficient weight gain, whereas the risks of LGA and macrosomia significantly increased with excessive weight gain regardless of pre-pregnancy BMI, except for macrosomia in women classified as overweight. The GWG cutoff points estimated by the adjusted AUC $>0.6$ corresponded to range $10-13.8 \mathrm{~kg}$ in underweight women; to range $10-13.7 \mathrm{~kg}$ in normal-weight women; to range $8.5-11.4 \mathrm{~kg}$ in overweight women; to range $5-13.3 \mathrm{~kg}$ in obese I women; and to be $4.7 \mathrm{~kg}$ for macrosomia in obese II women. When women with preterm birth were excluded, these cutoff points for GWG corresponded to range $10-12.6 \mathrm{~kg}$ in underweight women; to range $10-12.7 \mathrm{~kg}$ in normal-weight women; to range $11.3-11.4 \mathrm{~kg}$ in overweight women, range $11.5-14.1 \mathrm{~kg}$ in obese I women; and to $5.8 \mathrm{~kg}$ for macrosomia in obese II women. The optimal GWG ranges proposed by the present study are slightly higher than those recommended by the current Japanese guidelines: $9-12 \mathrm{~kg}$ for underweight and 7-12 kg for normal-weight women, and $\leq 5 \mathrm{~kg}$ (depending on individual cases) for overweight and obese women.

Several previous studies investigated optimal GWG for Asian women for whom there is no universal standard. The ranges proposed to date have been inconsistent (Fig. 1), with some proposing GWG below ${ }^{21,24-26}$ the IOM recommendations and others proposing GWG above the IOM range $\mathrm{e}^{25-27}$. These studies used various pregnancy outcomes and evaluation methods with various sample sizes of target populations differing in various respects (e.g., ethnicity, overweight/obese women, limited to full-term pregnancy). For example, Ee et al. ${ }^{26}$ investigated 1,529 women (63\%, Chinese) and estimated optimal GWG using composite perinatal outcome based on a combination of delivery method and fetal size for gestational age. Choi et al. ${ }^{27}$ investigated 3,285 Korean women with full-term singleton births and estimated optimal GWG as not exceeding a 5\% increase from the lowest total predicted risks of gestational hypertensive disorders, emergency cesarean section, and fetal size for gestational age. Among these previous studies, Morisaki et al. ${ }^{24}$ investigated the largest sample size of 104,070 women who were registered in 


\begin{tabular}{|c|c|c|c|c|c|c|c|c|}
\hline \multirow[b]{3}{*}{$\begin{array}{l}\text { Underweight women }(\mathrm{n}=18,382) \text { : } \\
\text { pre-pregnancy } \mathrm{BMI}<18.5\end{array}$} & \multicolumn{8}{|c|}{ Wegith gain during pregnancy recommended by Japanese Ministry of Health, Labour, and Welfare } \\
\hline & \multicolumn{3}{|c|}{$<9 \mathrm{~kg}(\mathrm{n}=6,417)$} & \multicolumn{2}{|l|}{$\begin{array}{l}9-12 \mathrm{~kg} \\
(\mathrm{n}=6,995)\end{array}$} & \multicolumn{3}{|c|}{$12<\operatorname{kg}(n=4,970)$} \\
\hline & n (\%) & Crude OR & $\begin{array}{l}\text { Adjusted OR } \\
(95 \% \mathrm{CI})\end{array}$ & n (\%) & OR & $\mathbf{n}(\%)$ & Crude OR & $\begin{array}{l}\text { Adjusted OR } \\
(95 \% \mathrm{CI})\end{array}$ \\
\hline SGA & $1172(18.3)$ & 1.86 & $1.87(1.69-2.07)^{\mathrm{a}}$ & $752(10.8)$ & 1 & $366(7.4)$ & 0.66 & $0.63(0.55-0.72)^{\mathrm{a}}$ \\
\hline Low birth weight & $2119(33.0)$ & 2.65 & $1.91(1.72-2.11)^{\mathrm{b}}$ & $1097(15.7)$ & 1 & $419(8.4)$ & 0.50 & $0.61(0.53-0.70)^{\mathrm{b}}$ \\
\hline Preterm birth & $1366(21.3)$ & 2.77 & $2.85(2.57-3.17)^{\mathrm{c}}$ & $623(8.9)$ & 1 & $216(4.4)$ & 0.47 & $0.43(0.37-0.51)^{c}$ \\
\hline LGA & $129(2.0)$ & 0.47 & $0.45(0.37-0.56)^{\mathrm{d}}$ & $290(4.2)$ & 1 & $466(9.4)$ & 2.39 & $2.48(2.13-2.89)^{\mathrm{d}}$ \\
\hline Macrosomia & $3(0.1)$ & 0.36 & $0.48(0.13-1.78)^{\mathrm{e}}$ & $9(0.1)$ & 1 & $33(0.7)$ & 5.19 & $4.64(2.21-9.78)^{\mathrm{e}}$ \\
\hline GDM & $281(4.4)$ & 1.94 & $1.89(1.55-2.30)^{\mathrm{f}}$ & $161(2.3)$ & 1 & $93(1.9)$ & 0.81 & $0.84(0.65-1.09)^{\mathrm{f}}$ \\
\hline Pregnancy-Induced Hypertension & $192(3.0)$ & 1.03 & $0.76(0.61-0.94)^{\mathrm{g}}$ & $203(2.9)$ & 1 & $218(4.4)$ & 1.54 & $1.71(1.40-2.09)^{\mathrm{g}}$ \\
\hline PPH with VD & $1021(15.9)$ & 0.73 & $0.85(0.78-0.94)^{\mathrm{d}}$ & $1434(20.5)$ & 1 & $1189(23.9)$ & 1.22 & $1.11(1.02-1.22)^{\mathrm{d}}$ \\
\hline PPH with CS & $302(4.7)$ & 0.98 & $0.66(0.56-0.78)^{\mathrm{e}}$ & $335(4.8)$ & 1 & $256(5.2)$ & 1.08 & $1.32(1.12-1.57)^{\mathrm{e}}$ \\
\hline \multirow{3}{*}{$\begin{array}{l}\text { Normal weight women } \\
(\mathrm{n}=61,960): \text { pre-pregnancy BMI } \\
18.5-22.9 \mathrm{~kg} / \mathrm{m}^{2}\end{array}$} & \multicolumn{8}{|c|}{ Weight gain during pregnancy recommended by Japanese Ministry of Health, Labour, and Welfare } \\
\hline & \multicolumn{3}{|c|}{$<7 \mathrm{~kg}(\mathrm{n}=10,686)$} & \multicolumn{2}{|l|}{$\begin{array}{l}7-12 \mathrm{~kg} \\
(\mathrm{n}=34,172)\end{array}$} & \multicolumn{3}{|c|}{$12<\operatorname{kg}(\mathrm{n}=17,102)$} \\
\hline & n (\%) & Crude OR & $\begin{array}{l}\text { Adjusted OR } \\
(95 \% \mathrm{CI})\end{array}$ & $\mathbf{n}(\%)$ & OR & $\mathbf{n}(\%)$ & Crude OR & \begin{tabular}{|l|} 
Adjusted OR \\
$(95 \% \mathrm{CI})$
\end{tabular} \\
\hline SGA & $1382(12.9)$ & 1.62 & $1.51(1.41-1.62)^{\mathrm{b}}$ & $2863(8.4)$ & 1.00 & $876(5.1)$ & 0.59 & $0.59(0.55-0.64)^{\mathrm{b}}$ \\
\hline Low birth weight & $3131(29.3)$ & 2.81 & $1.62(1.50-1.74)^{\mathrm{b}}$ & $4390(12.9)$ & 1.00 & $1114(6.5)$ & 0.47 & $0.62(0.57-0.68)^{\mathrm{b}}$ \\
\hline Preterm birth & $2490(23.3)$ & 3.17 & $3.16(2.98-3.36)^{c}$ & $2991(8.8)$ & 1.00 & $718(4.2)$ & 0.46 & $0.44(0.40-0.48)^{\mathrm{c}}$ \\
\hline LGA & $523(4.9)$ & 0.63 & $0.60(0.54-0.66)^{c}$ & $2598(7.6)$ & 1.00 & $2464(14.4)$ & 2.05 & $2.09(1.97-2.21)^{c}$ \\
\hline Macrosomia & $15(0.1)$ & 0.34 & $0.45(0.26-0.76)^{\mathrm{b}}$ & $139(0.4)$ & 1.00 & $235(1.4)$ & 3.41 & $2.72(2.20-3.37)^{\mathrm{b}}$ \\
\hline GDM & $755(7.1)$ & 2.17 & $2.08(1.89-2.29)^{\mathrm{f}}$ & $1159(3.4)$ & 1.00 & $408(2.4)$ & 0.7 & $0.72(0.64-0.81)^{\mathrm{f}}$ \\
\hline Pregnancy-Induced Hypertension & $476(4.5)$ & 1.20 & $0.70(0.62-0.78)^{g}$ & $1283(3.8)$ & 1.00 & $914(5.3)$ & 1.45 & $1.68(1.54-1.84)^{\mathrm{g}}$ \\
\hline PPH with VD & $1837(17.2)$ & 0.79 & $0.97(0.92-1.03)^{\mathrm{d}}$ & $7104(20.8)$ & 1.00 & $4215(24.7)$ & 1.25 & $1.11(1.07-1.16)^{\mathrm{d}}$ \\
\hline PPH with CS & $870(8.1)$ & 1.16 & $0.70(0.64-0.77)^{\mathrm{h}}$ & $2431(7.1)$ & 1.00 & $1304(7.6)$ & 1.08 & $1.36(1.26-1.46)^{\mathrm{h}}$ \\
\hline
\end{tabular}

Table 2. Risks of pregnancy outcomes for underweight and normal-weight women according to GWG below and above the recommendation of the Japanese government comparing GWG within the recommended levels. BMI, Body Mass Index; SGA, small for gestational age; LGA, large for gestational age; GDM, Gestational Diabetes Mellitus; PPH with VD, postpartum hemorrhage with vaginal delivery; PPH with CS, postpartum hemorrhage with cesarean section; GWG, gestational weight gain; adjusting for pre-pregnancy BMI, age, smoking, delivery method. ${ }^{\mathrm{b}}$ Adjusting for pre-pregnancy BMI, age, nulliparity, gestational week, smoking, delivery method. 'Adjusting for pre-pregnancy BMI, age, nulliparity, smoking, delivery method. ${ }^{\mathrm{d}}$ Adjusting for pre-pregnancy BMI, nulliparity, gestational week, smoking. ${ }^{e}$ Adjusting for pre-pregnancy BMI, age, gestational week. ${ }^{\mathrm{f}}$ Adjusting for pre-pregnancy BMI, age, smoking. ${ }^{\mathrm{g}}$ Adjusting for pre-pregnancy BMI, age, nulliparity, gestational week, delivery method. ${ }^{\mathrm{h}}$ Adjusting for pre-pregnancy BMI, age, nulliparity, gestational wek.

the Japanese perinatal database in 2005-2011, and developed a predictive model based on another sample of 1,283 mothers at a single hospital. The authors calculated expected the GWG over 40 weeks corresponding to a $1 \%$ increase in the risk of preterm delivery, SGA, complicated delivery, and preeclampsia. One study conducted by Hirooka-Nakama et al. ${ }^{25}$ used the same dataset as used in this study (similar in size to that used by Morisaki et al. ${ }^{24}$ ) but focused on overweight/obese women only and determined an optimal GWG of $0 \mathrm{~kg}$ based on the lowest risk probability of the sum of various pregnancy outcomes and $11.5 \mathrm{~kg}$, at which point the risk increased sharply. Although it may not be possible to take into account the speed of weight gain per trimester as ROC analysis is based on cross-sectional evaluation, calculating the adjusted cutoff points of individual multi-pregnancy outcomes is simple, straightforward, and easy to understand rather than composite outcomes or using complicated statistical models with various assumptions. We also adopted the cutoff points of GWG at the lower boundary of a $95 \%$ CI of AUC $>0.6$, and the results indicate that GWG has good predictive capability of a specific pregnancy outcome to determine accurate optimal GWG. Furthermore, we performed sensitivity analyses by limiting the subjects to those with full-term births because the database included large and tertiary hospitals and the subjects registered in the database might have had high risk profiles. If such unhealthy conditions were related to the birth of small babies, the true association might have been overestimated. The results were, however, similar between the models with inclusion or exclusion of preterm birth. Therefore, the results imply that optimal GWG is slightly higher than the current Japanese guidelines, regardless of the inclusion of preterm birth.

In this study, the AUCs had good predictive capability for macrosomia and LGA regardless of pre-pregnancy BMI category. Our ROC analyses showed that the optimal GWG range for large infants (i.e., LGA and macrosomia) exceeded the currently recommended threshold according to the Japanese guidelines (i.e., $5 \mathrm{~kg}$ for overweight and obese women). In this regard, the upper boundary of current GWG recommendations may still allow some room for these overweight/obese women to gain weight. In determining the upper boundary of GWG, the risk of hemorrhage at delivery is a major concern, as hemorrhagic obstetric complications are the leading cause of maternal deaths in Japan ${ }^{28}$. However, in the present study, PPH-VD and PPH-CS had low adjusted AUCs, 


\begin{tabular}{|c|c|c|c|c|c|c|}
\hline \multirow[b]{3}{*}{$\begin{array}{l}\text { Overweight women }(\mathrm{n}=9,985) \\
\text { pre-pregnancy BMI } 23-24.9 \mathrm{~kg} / \mathrm{m}^{2}\end{array}$} & \multicolumn{6}{|c|}{ Weight gain during pregnancy recommended by Japanese Ministry of Health, Labour, and Welfare } \\
\hline & \multicolumn{3}{|c|}{$\leq 5 \mathrm{~kg}(\mathrm{n}=1,506)$} & \multicolumn{3}{|c|}{$5<\operatorname{kg}(n=8,479)$} \\
\hline & n (\%) & Crude OR & \begin{tabular}{|l|} 
Adjusted OR (95\% \\
CI)
\end{tabular} & $\mathbf{n}(\%)$ & Crude OR & $\begin{array}{l}\text { Adjusted OR (95\% } \\
\text { CI) }\end{array}$ \\
\hline \multicolumn{7}{|c|}{ Risks associated with insufficient gestational weight gain } \\
\hline SGA & $195(13.0)$ & 2.3 & $2.05(1.70-2.46)^{\mathrm{a}}$ & $511(6.0)$ & 1 & - \\
\hline Low birth weight & $386(25.6)$ & 2.95 & $1.97(1.62-2.40)^{\mathrm{b}}$ & $886(10.5)$ & 1 & - \\
\hline Preterm birth & $326(21.7)$ & 2.77 & $2.84(2.45-3.29)^{\mathrm{c}}$ & $768(9.1)$ & 1 & - \\
\hline \multicolumn{7}{|c|}{ Risks associated with excess gestational weight gain } \\
\hline LGA & $114(7.6)$ & 1 & - & $1168(13.8)$ & 2.0 & $1.87(1.52-2.29)^{\mathrm{d}}$ \\
\hline Macrosomia & $6(0.4)$ & 1 & - & $96(1.1)$ & 2.86 & $2.18(0.95-5.00)^{\mathrm{a}}$ \\
\hline GDM & $183(12.2)$ & 1 & - & $511(6.0)$ & 0.46 & $0.49(0.41-0.59)^{\mathrm{e}}$ \\
\hline Pregnancy-Induced Hypertension & $98(6.5)$ & 1 & - & $576(6.8)$ & 1.05 & $1.50(1.17-1.91)^{\mathrm{d}}$ \\
\hline PPH with VD & $277(18.4)$ & 1 & - & $1863(22.0)$ & 1.25 & $1.01(0.87-1.17)^{\mathrm{f}}$ \\
\hline PPH with CS & $158(10.5)$ & 1 & - & $814(9.6)$ & 0.91 & $1.37(1.13-1.67)^{\mathrm{g}}$ \\
\hline \multirow{3}{*}{$\begin{array}{l}\text { Obese I }(\mathrm{n}=7,873) \text { : pre-pregnancy } \\
\text { BMI } 25-29.9 \mathrm{~kg} / \mathrm{m}^{2}\end{array}$} & \multicolumn{6}{|c|}{ Weight gain during pregnancy recommended by Japanese Ministry of Health, Labour, and Welfare } \\
\hline & \multicolumn{3}{|c|}{$\leq 5 \mathrm{~kg}(\mathrm{n}=2,236)$} & \multicolumn{3}{|c|}{$5<\operatorname{kg}(n=5,637)$} \\
\hline & n (\%) & Crude OR & \begin{tabular}{|l|}
$\begin{array}{l}\text { Adjusted OR } \\
(95 \% \mathrm{CI})\end{array}$ \\
\end{tabular} & $\mathbf{n}(\%)$ & Crude OR & $\begin{array}{l}\text { Adjusted OR } \\
(95 \% \mathrm{CI})\end{array}$ \\
\hline \multicolumn{7}{|c|}{ Risks associated with insufficient gestational weight gain } \\
\hline SGA & $222(9.9)$ & 1.77 & $1.55(1.29-1.87)^{\mathrm{h}}$ & $331(5.9)$ & 1 & - \\
\hline Low birth weight & $513(23.0)$ & 2.61 & $1.74(1.45-2.09)^{\mathrm{b}}$ & $578(10.3)$ & 1 & - \\
\hline Preterm birth & $416(18.6)$ & 2.35 & $2.45(2.12-2.83)^{\mathrm{i}}$ & $499(8.9)$ & 1 & - \\
\hline \multicolumn{7}{|c|}{ Risks associated with excess gestational weight gain } \\
\hline LGA & $225(10.1)$ & 1 & - & $1070(19.0)$ & 2.10 & $2.04(1.75-2.39)^{j}$ \\
\hline Macrosomia & $20(0.9)$ & 1 & - & $124(2.2)$ & 2.49 & $2.19(1.35-3.55)^{\mathrm{f}}$ \\
\hline GDM & $425(19.0)$ & 1 & - & $611(10.8)$ & 0.52 & $0.57(0.50-0.65)^{\mathrm{k}}$ \\
\hline Pregnancy-Induced Hypertension & $221(9.9)$ & 1 & - & $595(10.6)$ & 1.08 & $1.49(1.25-1.78)^{\mathrm{d}}$ \\
\hline PPH with VD & $410(18.3)$ & 1 & - & $1247(22.1)$ & 1.27 & $1.06(0.93-1.21)^{\mathrm{f}}$ \\
\hline PPH with CS & $262(11.7)$ & 1 & - & $648(11.5)$ & 0.98 & $1.28(1.09-1.51)^{\mathrm{b}}$ \\
\hline \multirow{3}{*}{$\begin{array}{l}\text { Obese II }(\mathrm{n}=3,136): \mathrm{pre-} \\
\text { pregnancy BMI } 30 \leq \mathrm{kg} / \mathrm{m}^{2}\end{array}$} & \multicolumn{6}{|c|}{ Weight gain during pregnancy recommended by Japanese Ministry of Health, Labour, and Welfare } \\
\hline & \multicolumn{3}{|c|}{$\leq 5 \mathrm{~kg}(\mathrm{n}=1,581)$} & \multicolumn{3}{|c|}{$>5 \mathrm{~kg}(\mathrm{n}=1,555)$} \\
\hline & n (\%) & Crude OR & \begin{tabular}{|l|} 
Adjusted OR (95\% \\
CI)
\end{tabular} & $\mathbf{n}(\%)$ & Crude OR & $\begin{array}{l}\text { Adjusted OR (95\% } \\
\text { CI) }\end{array}$ \\
\hline \multicolumn{7}{|c|}{ Risks associated with insufficient gestational weight gain } \\
\hline SGA & $145(9.2)$ & 1.84 & $1.66(1.25-2.22)^{1}$ & $81(5.2)$ & 1 & - \\
\hline Low birth weight & $277(17.5)$ & 2.04 & $1.53(1.15-2.05)^{\mathrm{f}}$ & $147(9.5)$ & 1 & - \\
\hline Preterm birth & $254(16.1)$ & 1.95 & $2.06(1.64-2.58)^{\mathrm{c}}$ & $139(8.9)$ & 1 & - \\
\hline \multicolumn{7}{|c|}{ Risks associated with excess gestational weight gain } \\
\hline LGA & $268(17.0)$ & 1 & - & $420(27.0)$ & 1.81 & $1.84(1.54-2.20)^{\mathrm{m}}$ \\
\hline Macrosomia & $30(1.9)$ & 1 & - & $72(4.6)$ & 2.51 & $2.40(1.54-3.75)^{\mathrm{a}}$ \\
\hline GDM & $470(29.7)$ & 1 & - & $331(21.3)$ & 0.64 & $0.69(0.58-0.81)^{\mathrm{e}}$ \\
\hline Pregnancy-Induced Hypertension & $245(15.5)$ & 1 & - & $256(16.5)$ & 1.08 & $1.29(1.05-1.58)^{\mathrm{d}}$ \\
\hline PPH with VD & $344(21.8)$ & 1 & - & $350(22.5)$ & 1.04 & $0.90(0.76-1.08)^{b}$ \\
\hline PPH with CS & $223(14.1)$ & 1 & - & $214(13.8)$ & 0.97 & $1.14(0.93-1.41)^{\mathrm{g}}$ \\
\hline
\end{tabular}

Table 3. Risks of pregnancy outcomes in overweight and obese women according to weight gain below or above $5 \mathrm{~kg}$. *Japanese Ministry of Labour, Health, and Welfare recommends up to $5 \mathrm{~kg}$ but depending on individual cases. BMI, Body Mass Index; SGA, small for gestational age; LGA, large for gestational age; GDM, Gestational Diabetes Mellitus; PPH with VD, postpartum hemorrhage with vaginal delivery; $\mathrm{PPH}$ with CS, postpartum

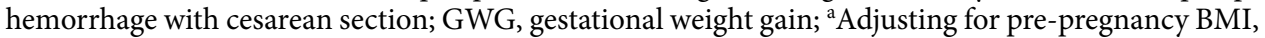
nulliparity, gestational week, delivery method. ${ }^{\mathrm{b}}$ Adjusting for pre-pregnancy BMI, age, nulliparity, gestational week. 'Adjusting for pre-pregnancy BMI, nulliparity, delivery method. ${ }^{\mathrm{d}}$ Adjusting for pre-pregnancy BMI, age, nulliparity, gestational week, delivery method. ${ }^{\mathrm{e} A d j u s t i n g}$ for pre-pregnancy BMI, age, smoking. ${ }^{\mathrm{f}}$ Adjusting for pre-pregnancy BMI, nulliparity, gestational week. ${ }^{\mathrm{g}}$ Adjusting for pre-pregnancy BMI, age, gestational week. ${ }_{\mathrm{h}}$ Adjusting for pre-pregnancy BMI, nulliparity, gestational week, smoking, delivery method. ${ }^{i}$ Adjusting for pre-pregnancy BMI, smoking, delivery method. ${ }^{j}$ Adjusting for pre-pregnancy BMI, gestational week, smoking, delivery method. ${ }^{k}$ Adjusting for pre-pregnancy BMI, age, nulliparity, smoking. ${ }^{1}$ Adjusting for pre-pregnancy BMI, gestational week. ${ }^{\mathrm{m}}$ Adjusting for pre-pregnancy BMI, gestational week, delivery method. 


\begin{tabular}{|c|c|c|c|c|c|c|c|c|c|c|c|c|c|}
\hline \multirow{3}{*}{$\begin{array}{l}\text { Asian specific BMI } \\
\text { categories }\end{array}$} & \multirow{3}{*}{\begin{tabular}{|l|} 
Outcome \\
SGA $^{\mathbf{a}}$ \\
\end{tabular}} & \multirow{3}{*}{$\begin{array}{c}\text { Crude } \\
\begin{array}{l}\text { GWG } \\
\text { cutoff }\end{array} \\
9.4\end{array}$} & \multicolumn{11}{|c|}{ Adjusted } \\
\hline & & & \multirow{2}{*}{\begin{tabular}{|l}
$\begin{array}{l}\text { GWG } \\
\text { cutoff }\end{array}$ \\
10 \\
\end{tabular}} & \multirow{2}{*}{\begin{tabular}{|l|}
$\begin{array}{l}\text { p-value of } \\
\text { continuous GWG }\end{array}$ \\
$<0.01$ \\
\end{tabular}} & \multirow{2}{*}{\begin{tabular}{|l|} 
AUC \\
0.63 \\
\end{tabular}} & \multicolumn{2}{|c|}{$95 \% \mathrm{CI}$} & \multirow{2}{*}{$\begin{array}{l}\text { Sensitivity } \\
0.58\end{array}$} & \multicolumn{2}{|c|}{ 95\% CI } & \multirow{2}{*}{\begin{tabular}{|l|} 
Specificity \\
0.62
\end{tabular}} & \multicolumn{2}{|c|}{$95 \% \mathrm{CI}$} \\
\hline & & & & & & 0.61 & 0.64 & & 0.56 & 0.60 & & 0.59 & 0.63 \\
\hline $\mathrm{BMI}<18.5 \mathrm{~kg} / \mathrm{m}^{2}$ & LBW $^{\mathrm{b}}$ & 9.1 & 11.6 & $<0.01$ & 0.61 & 0.59 & 0.62 & 0.56 & 0.53 & 0.58 & 0.60 & 0.57 & 0.63 \\
\hline \multirow{7}{*}{$(\mathrm{n}=18,382)$} & PTB $^{c}$ & 9.4 & 10 & $<0.01$ & 0.72 & \begin{tabular}{|l|l|}
0.71 \\
\end{tabular} & 0.73 & 0.66 & 0.64 & 0.68 & 0.67 & 0.64 & 0.68 \\
\hline & LGA $^{\mathrm{d}}$ & $\mid 11.1$ & 12 & $<0.01$ & 0.69 & 0.67 & 0.71 & 0.66 & 0.62 & 0.68 & 0.64 & 0.59 & 0.66 \\
\hline & Macrosomia $^{\mathrm{e}}$ & \begin{tabular}{|l|}
13.8 \\
\end{tabular} & 13.8 & $<0.01$ & 0.78 & 0.70 & 0.85 & 0.67 & 0.53 & 0.80 & 0.81 & 0.49 & 0.88 \\
\hline & $\mathrm{GDM}^{\mathrm{f}}$ & 8.7 & 8 & $<0.01$ & 0.61 & 0.58 & 0.63 & 0.42 & 0.38 & 0.46 & 0.75 & 0.70 & 0.78 \\
\hline & $\mathrm{PIH}^{\mathrm{g}}$ & 12 & 11.6 & $<0.01$ & 0.60 & 0.58 & 0.63 & 0.40 & 0.36 & 0.44 & 0.77 & 0.71 & 0.79 \\
\hline & PPHVD $^{\mathrm{h}}$ & \begin{tabular}{|l|}
10.4 \\
\end{tabular} & 10.3 & $<0.01$ & 0.53 & 0.52 & 0.54 & 0.54 & 0.52 & 0.56 & 0.50 & 0.48 & 0.52 \\
\hline & PPHCS $^{\mathrm{i}}$ & 15.5 & 10.5 & $<0.01$ & 0.60 & 0.58 & 0.62 & 0.65 & 0.61 & 0.68 & 0.50 & 0.46 & 0.52 \\
\hline Normal weight & $\mathrm{SGA}^{j}$ & 9.8 & 9.2 & $<0.01$ & 0.60 & 0.59 & 0.60 & 0.54 & 0.52 & 0.55 & 0.61 & 0.59 & 0.62 \\
\hline $18.5-23 \mathrm{~kg} / \mathrm{m}^{2}$ & LBW $^{\mathrm{b}}$ & 9.1 & 11.5 & $<0.01$ & 0.57 & 0.56 & 0.58 & 0.55 & 0.53 & 0.56 & 0.56 & 0.55 & 0.57 \\
\hline \multirow{7}{*}{$(\mathrm{n}=61,960)$} & PTB $^{\mathrm{c}}$ & 8.3 & 10 & $<0.01$ & 0.70 & 0.69 & 0.71 & 0.59 & 0.57 & 0.60 & 0.71 & 0.70 & 0.73 \\
\hline & LGA $^{\mathbf{c}}$ & \begin{tabular}{|l|}
10.9 \\
\end{tabular} & 11.7 & $<0.01$ & 0.64 & 0.63 & 0.64 & 0.51 & 0.50 & 0.52 & 0.69 & 0.67 & 0.70 \\
\hline & Macrosomia $^{\mathrm{b}}$ & 11.3 & 13.7 & $<0.01$ & 0.70 & 0.67 & 0.72 & 0.61 & 0.56 & 0.66 & 0.69 & 0.63 & 0.73 \\
\hline & $\mathrm{GDM}^{\mathrm{f}}$ & 7.8 & 8.5 & $<0.01$ & 0.61 & 0.59 & 0.62 & 0.44 & 0.42 & 0.46 & 0.73 & 0.71 & 0.74 \\
\hline & $\mathrm{PIH}^{\mathrm{g}}$ & 12.6 & 13.3 & $<0.01$ & 0.60 & 0.59 & 0.61 & 0.44 & 0.42 & 0.45 & 0.72 & 0.69 & 0.74 \\
\hline & PPHVD $^{\mathrm{h}}$ & 10.4 & 10.8 & $<0.01$ & 0.52 & 0.51 & 0.52 & 0.40 & 0.39 & 0.41 & 0.63 & 0.61 & 0.63 \\
\hline & PPHCS $^{k}$ & 6.8 & 10.3 & $<0.01$ & 0.57 & 0.56 & 0.58 & 0.55 & 0.53 & 0.56 & 0.56 & 0.54 & 0.57 \\
\hline Overweight & $\mathrm{SGA}^{1}$ & 7.3 & 9.7 & $<0.01$ & 0.60 & 0.58 & 0.62 & 0.54 & 0.50 & 0.58 & 0.61 & 0.57 & 0.64 \\
\hline $23-24.9 \mathrm{~kg} / \mathrm{m}^{2}$ & LBW $^{\mathrm{k}}$ & 6.1 & 7.3 & $<0.01$ & 0.59 & 0.56 & 0.61 & 0.59 & 0.55 & 0.63 & 0.53 & 0.48 & 0.57 \\
\hline \multirow{7}{*}{$(\mathrm{n}=9,985)$} & PTB $^{\mathrm{m}}$ & 6.2 & 8.5 & $<0.01$ & 0.65 & 0.64 & 0.67 & \begin{tabular}{|l|}
0.58 \\
\end{tabular} & 0.54 & 0.61 & 0.66 & 0.62 & 0.69 \\
\hline & LGA $^{\mathbf{n}}$ & 8.9 & 11.3 & $<0.01$ & 0.62 & 0.61 & 0.64 & 0.55 & 0.52 & 0.58 & 0.63 & 0.59 & 0.65 \\
\hline & Macrosomia $^{\mathrm{g}}$ & 10.3 & \begin{tabular}{|l|}
11.4 \\
\end{tabular} & $<0.01$ & 0.66 & 0.61 & 0.71 & 0.72 & 0.63 & 0.79 & 0.54 & 0.42 & 0.60 \\
\hline & $\mathrm{GDM}^{\mathrm{f}}$ & 7.1 & 8.1 & $<0.01$ & 0.58 & 0.56 & 0.61 & 0.55 & 0.51 & 0.59 & 0.60 & 0.55 & 0.64 \\
\hline & $\mathrm{PIH}^{\mathrm{g}}$ & 10 & 11 & $<0.01$ & 0.58 & 0.55 & 0.60 & 0.50 & 0.46 & 0.54 & 0.62 & 0.58 & 0.65 \\
\hline & PPHVD $^{\circ}$ & 6.4 & 14.5 & $<0.01$ & 0.51 & 0.50 & 0.53 & 0.16 & 0.14 & 0.18 & 0.87 & 0.84 & 0.88 \\
\hline & PPHCS $^{\mathrm{i}}$ & 6 & 9.5 & $<0.01$ & 0.55 & 0.52 & 0.56 & 0.57 & 0.52 & 0.60 & 0.52 & 0.48 & 0.54 \\
\hline Obese I & SGAj & 3.2 & 6 & $<0.01$ & 0.57 & 0.54 & 0.59 & 0.32 & 0.27 & 0.36 & 0.80 & 0.73 & 0.82 \\
\hline $25-29.9 \mathrm{~kg} / \mathrm{m}^{2}$ & LBW $^{e}$ & 3.1 & 7.7 & $<0.01$ & 0.60 & 0.57 & 0.63 & 0.51 & 0.45 & 0.55 & 0.65 & 0.59 & 0.68 \\
\hline \multirow{7}{*}{$(\mathrm{n}=7,873)$} & PTB $^{\mathbf{P}}$ & 5.3 & 5 & $<0.01$ & 0.63 & 0.61 & 0.65 & 0.51 & 0.48 & 0.55 & 0.71 & 0.66 & 0.73 \\
\hline & LGA $^{q}$ & 8.5 & 8 & $<0.01$ & 0.62 & 0.60 & 0.63 & \begin{tabular}{|l|}
0.54 \\
\end{tabular} & 0.50 & 0.56 & 0.64 & 0.60 & 0.66 \\
\hline & Macrosomia $^{\mathrm{k}}$ & 6.8 & 13.3 & $<0.01$ & 0.65 & 0.60 & 0.69 & 0.39 & 0.30 & \begin{tabular}{|l|}
0.47 \\
\end{tabular} & 0.84 & 0.75 & $\begin{array}{l}0.88 \\
\end{array}$ \\
\hline & $\mathrm{GDM}^{\mathrm{n}}$ & 2.6 & 5.6 & $<0.01$ & 0.58 & 0.56 & 0.60 & 0.44 & 0.40 & 0.47 & 0.69 & 0.66 & 0.73 \\
\hline & $\mathrm{PIH}^{\mathrm{g}}$ & 7 & 12 & $<0.01$ & 0.59 & 0.57 & 0.61 & 0.37 & 0.33 & 0.41 & 0.77 & 0.73 & 0.79 \\
\hline & PPHVD $^{\circ}$ & 9.6 & 9.1 & $<0.01$ & 0.51 & 0.50 & 0.53 & 0.46 & 0.43 & \begin{tabular}{|l|}
0.48 \\
\end{tabular} & 0.57 & 0.54 & 0.60 \\
\hline & PPHCS $^{k}$ & 8.5 & 7.2 & $<0.01$ & 0.54 & 0.52 & 0.56 & 0.58 & 0.54 & 0.62 & 0.49 & 0.45 & 0.52 \\
\hline Obese II & $\mathrm{SGA}^{\mathrm{r}}$ & 3.2 & 4.1 & $<0.01$ & 0.58 & 0.54 & 0.62 & 0.53 & 0.45 & \begin{tabular}{|l|}
0.59 \\
\end{tabular} & 0.61 & 0.52 & 0.66 \\
\hline $30 \leq \mathrm{kg} / \mathrm{m}^{2}$ & LBW $^{\circ}$ & 3.1 & 3 & $<0.01$ & \begin{tabular}{|l|}
0.57 \\
\end{tabular} & 0.52 & 0.61 & 0.46 & 0.38 & 0.53 & 0.64 & 0.54 & 0.71 \\
\hline \multirow{7}{*}{$(\mathrm{n}=3,136)$} & PTB $^{\mathrm{s}}$ & 5.1 & 4.5 & $<0.01$ & 0.61 & 0.58 & 0.64 & 0.56 & 0.49 & 0.60 & 0.63 & 0.55 & 0.66 \\
\hline & LGA $^{\mathrm{r}}$ & 8.5 & 3 & $<0.01$ & 0.61 & 0.59 & 0.64 & 0.75 & 0.71 & \begin{tabular}{|l|}
0.78 \\
\end{tabular} & 0.42 & 0.36 & 0.45 \\
\hline & Macrosomia $^{1}$ & 6.8 & 4.7 & $<0.01$ & 0.67 & 0.62 & 0.72 & 0.81 & 0.70 & 0.87 & 0.44 & 0.33 & 0.48 \\
\hline & GDM $^{f}$ & 2.6 & 6.4 & $<0.01$ & 0.56 & 0.54 & 0.59 & 0.51 & 0.46 & 0.55 & 0.60 & 0.55 & 0.63 \\
\hline & $\mathrm{PIH}^{\mathrm{g}}$ & 7 & 11.2 & $<0.01$ & 0.55 & 0.52 & 0.58 & 0.31 & 0.25 & 0.35 & 0.80 & 0.74 & 0.82 \\
\hline & PPHVD $^{\mathrm{e}}$ & 9.6 & 6 & 0.10 & 0.52 & 0.49 & 0.55 & 0.54 & 0.49 & 0.58 & 0.51 & 0.45 & 0.54 \\
\hline & PPHCS $^{e}$ & 8.5 & 5 & 0.00 & 0.54 & 0.51 & 0.57 & 0.63 & 0.57 & \begin{tabular}{|l|}
0.67 \\
\end{tabular} & 0.45 & 0.37 & 0.49 \\
\hline
\end{tabular}

Table 4. Gestational weight gain cutoff points based on maximized area under curve according to pregnancy outcomes. AUC, Area under curve, BMI, Body Mass Index; SGA, small for gestational age; LGA, large for gestational age; LBW, low birth weight; PTB, preterm birth; GDM, Gestational Diabetes Mellitus; PIH, pregnancy-induced hypertension; $\mathrm{PPH}$ with VD, postpartum hemorrhage with vaginal delivery; $\mathrm{PPH}$ with CS, postpartum hemorrhage with cesarean section; GWG, gestational weight gain, 95\%CI, 95\%confidence Interval. a Adjusting for pre-pregnancy BMI, age, gestational week, smoking, delivery method. ${ }^{\mathrm{b}}$ Adjusting for pre-pregnancy BMI, age, nulliparity, gestational week, smoking, delivery method. ${ }^{c}$ Adjusting for prepregnancy BMI, age, nulliparity, smoking, delivery method. ${ }^{\mathrm{d}}$ Adjusting for pre-pregnancy BMI, age, nulliparity, gestational week, smoking. ${ }^{\mathrm{e}}$ Adjusting for pre-pregnancy BMI, age, gestational week, nulliparity. ${ }^{\mathrm{f}}$ Adjusting for pre-pregnancy BMI, age, smoking. ${ }^{\mathrm{g} A d j u s t i n g}$ for pre-pregnancy BMI, age, nulliparity, gestational week, delivery method. ${ }^{\mathrm{h}}$ Adjusting for pre-pregnancy BMI, smoking, nulliparity, gestational week. ${ }^{\mathrm{i}}$ Adjusting for 
pre-pregnancy BMI, age, gestational week. ${ }^{j}$ Adjusting for pre-pregnancy BMI, nulliparity, gestational week, smoking, delivery method. ${ }^{\mathrm{k}}$ Adjusting for pre-pregnancy BMI, age, nulliparity, gestational week. ${ }^{1}$ Adjusting for pre-pregnancy BMI, nulliparity, gestational week, delivery method. ${ }^{\mathrm{m}}$ Adjusting for pre-pregnancy BMI, nulliparity, smoking, delivery method. ${ }^{\text {n} A d j u s t i n g ~ f o r ~ p r e-p r e g n a n c y ~ B M I, ~ a g e, ~ n u l l i p a r i t y, ~ s m o k i n g . ~}{ }^{\circ}$ Adjusting

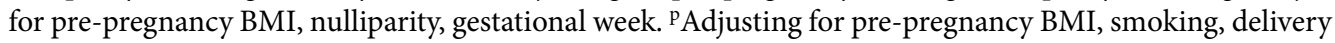
method. ${ }^{\mathrm{q} A d j u s t i n g}$ for pre-pregnancy BMI, age, gestational week, smoking. ${ }^{\mathrm{r}}$ Adjusting for pre-pregnancy BMI,

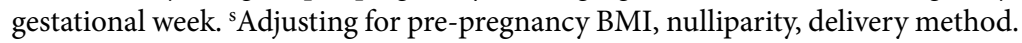

sensitivity, and specificity, implying that weight gain may have little effect on hemorrhage. Our results are consistent with a previous study ${ }^{29}$ indicating that overweight/obese women are more likely to have high incidences of LGA, GDM, and PIH, but these pregnancy outcomes/complications are independent of weight gain, and are therefore difficult to manage by weight gain control. In Japan, obese women are more likely to receive strict instruction to limit weight gain to prevent dystocia $^{30}$, but they might feel more at ease if the upper boundary of the current Japanese guidelines is increased for overweight and obese women (i.e., $\sim 30 \mathrm{~kg} / \mathrm{m}^{2}$ as implied by the present study in Fig. 1).

We expected that excessive GWG would be associated with an increased risk of GDM, but found the opposite: underweight and normal-weight women with GWG below the recommendations were more likely to have GDM, whereas normal-weight, overweight, and obese women with GWG above the recommendations were less likely to have GDM. These findings are consistent with a previous study ${ }^{2}$ using the same dataset, indicating that there were no errors in our calculations. One explanation may be that GDM is usually diagnosed in the second trimester (i.e., around gestational week 24-28), and it is therefore possible that women with a diagnosis of GDM are monitored more closely and encouraged to improve their behaviors (i.e., diet, physical activity) resulting in limited total GWG. Similarly, two Asian studies of 48,867 Chinese women ${ }^{31}$ and 7,843 Korean women ${ }^{17}$ provide $^{2}$ further examples of a counterintuitive relationship between GDM and GWG during pregnancy. The subjects in these studies were recruited at large hospitals, where individual mothers might have had more health concerns, and therefore overweight and obese women in particular were more likely to adhere to weight-gain recommendations. Furthermore, one study compared 4,930 Asian Indians with 2,868 Caucasians and demonstrated that the prevalence of diabetes in underweight subjects was higher in Asians than in Caucasians. Ethnic differences in diabetes susceptibility (Asians $>$ Caucasians) ${ }^{32}$ may also enhance the attention of individuals to strict blood-sugar control during pregnancy.

This study had some limitations. First, due to the database used in this study, all of the variables had some degree of unrealistic data (e.g., maternal height outside the range of 60-200 cm or pre-pregnancy BMI), implying that data reliability may have been low. Although these corresponded to a total of 1,146 subjects constituting $<1 \%$ of all data, thus reducing their impact on the results, they were excluded to increase the accuracy of the analyses. Second, data on lifestyle factors, including smoking and drinking habits, as well as social factors, including socioeconomic status, were missing. The birth of small babies has been linked to maternal smoking ${ }^{33}$ and poor nutritional status ${ }^{34}$; therefore, it would have been better to adjust for social and environmental factors. Third, pre-pregnancy BMI and GWG were estimated based on self-reporting of weight before pregnancy, which might have been subject to recall bias. Fourth, in this study, we did not investigate preeclampsia as an outcome; however, this exclusion is recognized by the IOM Working Group as there is only weak evidence that excessive weight gain can cause preeclampsia and the reverse association is also plausible, in that edema due to preeclampsia may cause increased weight gain ${ }^{35}$. Finally, the majority of women registered in the JSOG birth database had delivered at tertiary hospitals, indicating that they were more likely to represent a high-risk group. Although we confirmed that the results did not change if we excluded those with preterm birth, further research is needed to assess whether our findings are generalizable to other Japanese populations.

Despite these limitations, the present study based on adjusted ROC curves indicated that the current Japanese recommendations for GWG may be lower than the optimal GWG ranges and might have a merit if both lower and upper boundaries are increased.

\section{Methods}

Subjects for analyses in the JSOG database. JSOG developed the Successive Pregnancy Birth Registry System in 2001 to monitor the safety of pregnancy outcomes. This cross-sectional study was conducted after approval from the Ethics Committee of Hamamatsu University School of Medicine. The patient records were anonymized and de-identified prior to analysis and the committee confirmed that the research was performed in accordance with relevant guidelines. Informed consent was obtained in the form of opting out on the website at http://www.jsog.or.jp/activity/pdf/Clinical_research_2017-69.pdf. The details have been described in a previous study ${ }^{2}$. Weight gain during pregnancy (i.e., GWG) was calculated by subtracting pre-pregnancy weight from maternal weight at delivery. Briefly, we analyzed 186,235 women who gave birth at gestational week 22 or later between January 1 and December 31, 2013 in approximately 280 secondary and tertiary hospitals. Figure 2 is the flowchart of subjects included in the analyses. After excluding multiple pregnancies $(n=12,533)$, congenital anomalies $(n=2,763)$, underlying illnesses $(n=30,173)$, and post-term births $(n=461)$, the dataset comprised 140,701 women with full-term singleton babies. Among these, missing data on SGA $(n=50)$, LGA $(n=132)$, pre-pregnancy BMI $(n=20,683)$, age $(n=939)$, infant sex $(n=113)$, gestational weeks $(n=92)$, GWG $(n=31,190)$, smoking status $(n=6,144)$, and hemorrhage $(n=1,413)$ were excluded. We further excluded unrealistic data, including cases with recorded birth weight $<500 \mathrm{~g}(n=211)$, birth height $<25 \mathrm{~cm}(n=176)$, if mother's maternal weight was outside the range $20-200 \mathrm{~kg}(n=105)$, if mother's maternal height was outside the range $60-200 \mathrm{~cm}(n=156)$, if pre-pregnancy BMI was outside the range $6-50 \mathrm{~kg} / \mathrm{m}^{2}(n=254), \mathrm{GWG}<-8 \mathrm{~kg}$ or $>40 \mathrm{~kg}$ 


\begin{tabular}{|c|c|c|c|c|c|c|c|c|c|c|c|c|c|}
\hline \multirow{3}{*}{$\begin{array}{l}\text { Asian specific BMI } \\
\text { categories }\end{array}$} & \multirow{3}{*}{\begin{tabular}{|l|} 
Outcome \\
SGA $^{\mathrm{a}}$ \\
\end{tabular}} & \multirow{3}{*}{$\begin{array}{l}\text { Crude } \\
\begin{array}{l}\text { GWG } \\
\text { cutoff }\end{array} \\
9.4\end{array}$} & \multicolumn{11}{|c|}{ Adjusted } \\
\hline & & & \multirow{2}{*}{$\begin{array}{l}\text { GWG } \\
\text { cutoff }\end{array}$} & \multirow{2}{*}{$\begin{array}{l}\text { p-value of } \\
\text { continuous } \\
\text { GWG } \\
<0.01 \\
\end{array}$} & \multirow{2}{*}{\begin{tabular}{|l|} 
AUC \\
0.62 \\
\end{tabular}} & \multicolumn{2}{|c|}{$95 \% \mathrm{CI}$} & \multirow{2}{*}{$\begin{array}{l}\text { Sensitivity } \\
0.55\end{array}$} & \multicolumn{2}{|c|}{$95 \% \mathrm{CI}$} & \multirow{2}{*}{$\begin{array}{l}\text { Specificity } \\
0.64\end{array}$} & \multicolumn{2}{|c|}{$95 \%$ CI } \\
\hline & & & & & & 0.62 & 0.63 & & 0.53 & 0.56 & & 0.62 & 0.65 \\
\hline $\mathrm{BMI}<18.5 \mathrm{~kg} / \mathrm{m}^{2}$ & LBW $^{\mathbf{a}}$ & 9.5 & 10 & $<0.01$ & 0.62 & 0.61 & 0.63 & 0.54 & 0.52 & 0.55 & 0.65 & 0.63 & 0.66 \\
\hline \multirow{2}{*}{$(\mathrm{n}=16,177)$} & LGA $^{\mathbf{b}}$ & 11.5 & 12.6 & $<0.01$ & 0.64 & 0.63 & 0.65 & 0.54 & 0.53 & 0.55 & 0.67 & 0.66 & 0.68 \\
\hline & Macrosomia $^{c}$ & 13 & 12 & $<0.01$ & 0.69 & 0.67 & 0.71 & 0.56 & 0.52 & 0.59 & 0.73 & 0.69 & 0.76 \\
\hline Normal weight & SGA $^{\mathrm{d}}$ & 9.7 & 10.6 & $<0.01$ & 0.62 & 0.61 & 0.62 & 0.53 & 0.51 & 0.55 & 0.64 & 0.62 & 0.66 \\
\hline $18.5-23 \mathrm{~kg} / \mathrm{m}^{2}$ & LBW $^{\mathbf{a}}$ & 9.5 & 10.4 & $<0.01$ & 0.61 & 0.60 & 0.62 & 0.63 & 0.61 & 0.65 & 0.54 & 0.51 & 0.55 \\
\hline \multirow{2}{*}{$(\mathrm{n}=55,761)$} & LGA $^{\mathrm{a}}$ & 10.9 & 10 & $<0.01$ & 0.61 & 0.60 & 0.62 & 0.63 & 0.61 & 0.65 & 0.54 & 0.51 & 0.55 \\
\hline & Macrosomia $^{a}$ & 11.3 & 12.7 & $<0.01$ & 0.70 & 0.67 & 0.72 & 0.61 & 0.56 & 0.66 & 0.69 & 0.64 & 0.72 \\
\hline Overweight & $\mathrm{SGA}^{\mathrm{e}}$ & 9.7 & 10 & $<0.01$ & 0.62 & 0.59 & 0.64 & 0.66 & 0.61 & 0.70 & 0.52 & 0.47 & 0.55 \\
\hline $23-24.9 \mathrm{~kg} / \mathrm{m}^{2}$ & LBW $^{f}$ & 9.9 & 7.5 & $<0.01$ & 0.61 & 0.58 & 0.63 & 0.40 & 0.35 & 0.45 & 0.74 & 0.68 & 0.78 \\
\hline \multirow{2}{*}{$(\mathrm{n}=8,891)$} & LGA $^{\mathbf{g}}$ & 10.6 & 11.3 & $<0.01$ & 0.62 & 0.61 & 0.64 & 0.55 & 0.52 & 0.58 & 0.63 & 0.59 & 0.66 \\
\hline & Macrosomia $^{\mathrm{h}}$ & 11.4 & 11.4 & $<0.01$ & 0.66 & 0.61 & 0.72 & 0.73 & 0.61 & 0.79 & 0.54 & 0.42 & 0.59 \\
\hline Obese I & $\mathrm{SGA}^{\mathrm{i}}$ & 3.5 & 7 & $<0.01$ & 0.59 & 0.56 & 0.62 & 0.53 & 0.47 & 0.58 & 0.61 & 0.53 & 0.65 \\
\hline $25-29.9 \mathrm{~kg} / \mathrm{m}^{2}$ & LBW $^{\mathrm{c}}$ & 5.4 & 7.5 & $<0.01$ & 0.60 & 0.57 & 0.63 & 0.53 & 0.47 & 0.57 & 0.63 & 0.57 & 0.67 \\
\hline \multirow{2}{*}{$(\mathrm{n}=6,958)$} & LGA $^{\mathbf{g}}$ & 8.9 & 11.5 & $<0.01$ & 0.62 & 0.60 & 0.64 & 0.42 & 0.39 & 0.45 & 0.75 & 0.71 & 0.77 \\
\hline & Macrosomia $^{\mathrm{c}}$ & 10.3 & 14.1 & $<0.01$ & 0.65 & 0.61 & 0.70 & 0.40 & 0.30 & 0.47 & 0.85 & 0.75 & 0.88 \\
\hline Obese II & $\mathrm{SGA}^{j}$ & 3.1 & 3.4 & $<0.01$ & 0.59 & 0.54 & 0.64 & 0.45 & 0.34 & 0.53 & 0.72 & 0.61 & 0.76 \\
\hline $30 \leq \mathrm{kg} / \mathrm{m}^{2}$ & LBW $^{f}$ & 1.4 & 3.5 & $<0.01$ & 0.61 & 0.56 & 0.65 & 0.54 & 0.45 & 0.62 & 0.66 & 0.52 & 0.72 \\
\hline \multirow{2}{*}{$(\mathrm{n}=2,743)$} & LGA $^{\mathrm{k}}$ & 8.5 & 5 & $<0.01$ & 0.61 & 0.59 & 0.64 & 0.74 & 0.69 & 0.77 & 0.43 & 0.39 & 0.47 \\
\hline & Macrosomia $^{1}$ & 6.8 & 5.8 & $<0.01$ & 0.66 & 0.60 & 0.71 & \begin{tabular}{|l|}
0.81 \\
\end{tabular} & 0.68 & 0.87 & 0.43 & \begin{tabular}{|l|l|}
0.33 \\
\end{tabular} & 0.48 \\
\hline
\end{tabular}

Table 5. Gestational weight gain cutoff points according to SGA, LBW, LGA, and macrosomia excluding women with preterm birth. AUC, Area under curve, BMI, Body Mass Index; SGA, small for gestational age; LGA, large for gestational age; LBW, low birth weight; GWG, gestational weight gain, 95\%CI, 95\%Confidence Interval. aAdjusting for pre-pregnancy BMI, age, nulliparity, gestational week, smoking, delivery method. ${ }^{\mathrm{b}}$ Adjusting for pre-pregnancy BMI, age, smoking, nulliparity. ${ }^{\mathrm{C}}$ Adjusting for pre-pregnancy BMI, age, nulliparity, gestational week. ${ }^{\mathrm{d} A d j u s t i n g}$ for pre-pregnancy BMI, age, gestational week, smoking, delivery method. ${ }^{\mathrm{e} A d j u s t i n g ~ f o r ~ p r e-p r e g n a n c y ~ B M I, ~ n u l l i p a r i t y . ~}{ }^{\mathrm{f} A d j u s t i n g ~ f o r ~ p r e-p r e g n a n c y ~ B M I, ~ n u l l i p a r i t y, ~}$ gestational week. ${ }^{\mathrm{g}}$ Adjusting for pre-pregnancy BMI, age, smoking, delivery method. ${ }^{\mathrm{h}}$ Adjusting for prepregnancy BMI, age, nulliparity, gestational week, delivery method. ${ }^{i}$ Adjusting for pre-pregnancy BMI, nulliparity, smoking. ${ }^{j}$ Adjusting for pre-pregnancy BMI. ${ }^{k}$ Adjusting for pre-pregnancy BMI, age. ${ }^{1}$ Adjusting for pre-pregnancy BMI, nulliparity, gestational week, delivery method.

\begin{tabular}{|c|c|c|c|c|c|}
\hline & Underweight & Normal-weight & Overweight & Obese I & Obese II \\
\hline Asian Specific BMI, kg/m² & $<18.5$ & $18.5-22.9$ & $23-24.9$ & $25-29.9$ & $30 \leq$ \\
\hline \multicolumn{6}{|l|}{ Conventional guideline } \\
\hline Japanese Ministry guideline & $9-12 \mathrm{~kg}$ & $7-12 \mathrm{~kg}$ & $7-12 \mathrm{~kg}$ & $5 \mathrm{~kg}^{*}$ & $5 \mathrm{~kg}^{*}$ \\
\hline Institute Of Medicine & $12.5-18 \mathrm{~kg}$ & $11.5-16 \mathrm{~kg}$ & $11.5-16 \mathrm{~kg}$ & $7-11.5 \mathrm{~kg}$ & $5.0-9.0 \mathrm{~kg}$ \\
\hline \multicolumn{6}{|l|}{ The present study } \\
\hline \multicolumn{6}{|l|}{ Women with singleton baby } \\
\hline SGA, LBW, PTB & $10 \mathrm{~kg}$ for SGA and PTB & $10 \mathrm{~kg}$ for PTB & $8.5 \mathrm{~kg}$ for PTB & $5 \mathrm{~kg}$ for PTB & - \\
\hline LGA and Macrosomia & $12 \mathrm{~kg}$ and $13.8 \mathrm{~kg}$ & $11.7 \mathrm{~kg}$ and $13.7 \mathrm{~kg}$ & $11.3 \mathrm{~kg}$ and $11.4 \mathrm{~kg}$ & $8 \mathrm{~kg}$ and $13.3 \mathrm{~kg}$ & $4.7 \mathrm{~kg}$ for Macrosomia \\
\hline \multicolumn{6}{|l|}{ Women excluding preterm birth } \\
\hline SGA and LBW & $11.2 \mathrm{~kg}$ and $10 \mathrm{~kg}$ & $10.6 \mathrm{~kg}$ and $10.4 \mathrm{~kg}$ & - & - & - \\
\hline LGA and Macrosomia & $12.6 \mathrm{~kg}$ and $12 \mathrm{~kg}$ & $10 \mathrm{~kg}$ and $12.7 \mathrm{~kg}$ & $11.3 \mathrm{~kg}$ and $11.4 \mathrm{~kg}$ & $11.5 \mathrm{~kg}$ and $14.1 \mathrm{~kg}$ & $5.8 \mathrm{~kg}$ for Macrosomia \\
\hline \multicolumn{6}{|c|}{ Suggested range by previous studies that specifically target Asian women } \\
\hline Morisaki, et al. (2017) & $12.2(10.8-13.6)$ & $\begin{array}{c}10.9(9.5-12.4) \text { for BMI } 18.5-19.9 \\
9.9(8.4-11.4) \text { for BMI } 20-22.9\end{array}$ & $7.7(5.8-9.6)$ & $7.7(5.8$ - 9.6) for BMI $25-27.4$ & - \\
\hline Hirooka-Nakama, et al. (2018) & - & - & - & $0-11.5 \mathrm{~kg}$ & $-7 \mathrm{~kg}$ \\
\hline Nomura, et al. (2017) & - & $9.7-10.4$ & $9.7-10.4$ & - & - \\
\hline Choi, et al. (2017) & $20.8(16.7-24.7)$ & $16.6(11.5-21.5)$ & $13.1(8.0-17.7)$ & $14.4(7.5-21.9)$ & $14.4(7.5-21.9)$ \\
\hline Ee, et al. (2014) & $19.5(12.9-23.9)$ & $13.7(7.7-18.8)$ & $7.9(2.6-14.0)$ & $1.8(-5.0-7.0)$ & $1.8(-5.0-7.0)$ \\
\hline
\end{tabular}

Figure 1. Weight gain during pregnancy recommended by the Institute of Medicine, the Japanese Ministry of Health, Labour, and Welfare, present and previous studies in Asian countries. *Approximately up to $5 \mathrm{~kg}$ but depending on individual cases BMI, Body Mass Index; SGA, small for gestational age; LGA, large for gestational age; LBW, low birth weight; PTB, preterm birth.

$(n=767)$, parity number exceeding $30(n=2)$, and infant head circumference exceeding $66.5 \mathrm{~cm}(n=122)$. A total of 1,146 subjects showed such unrealistic data. Ultimately, 101,336 subjects were included in our analyses. In 2000, the WHO redefined the BMI classification for the Asian population ${ }^{22}$, proposing the criterion for obesity 


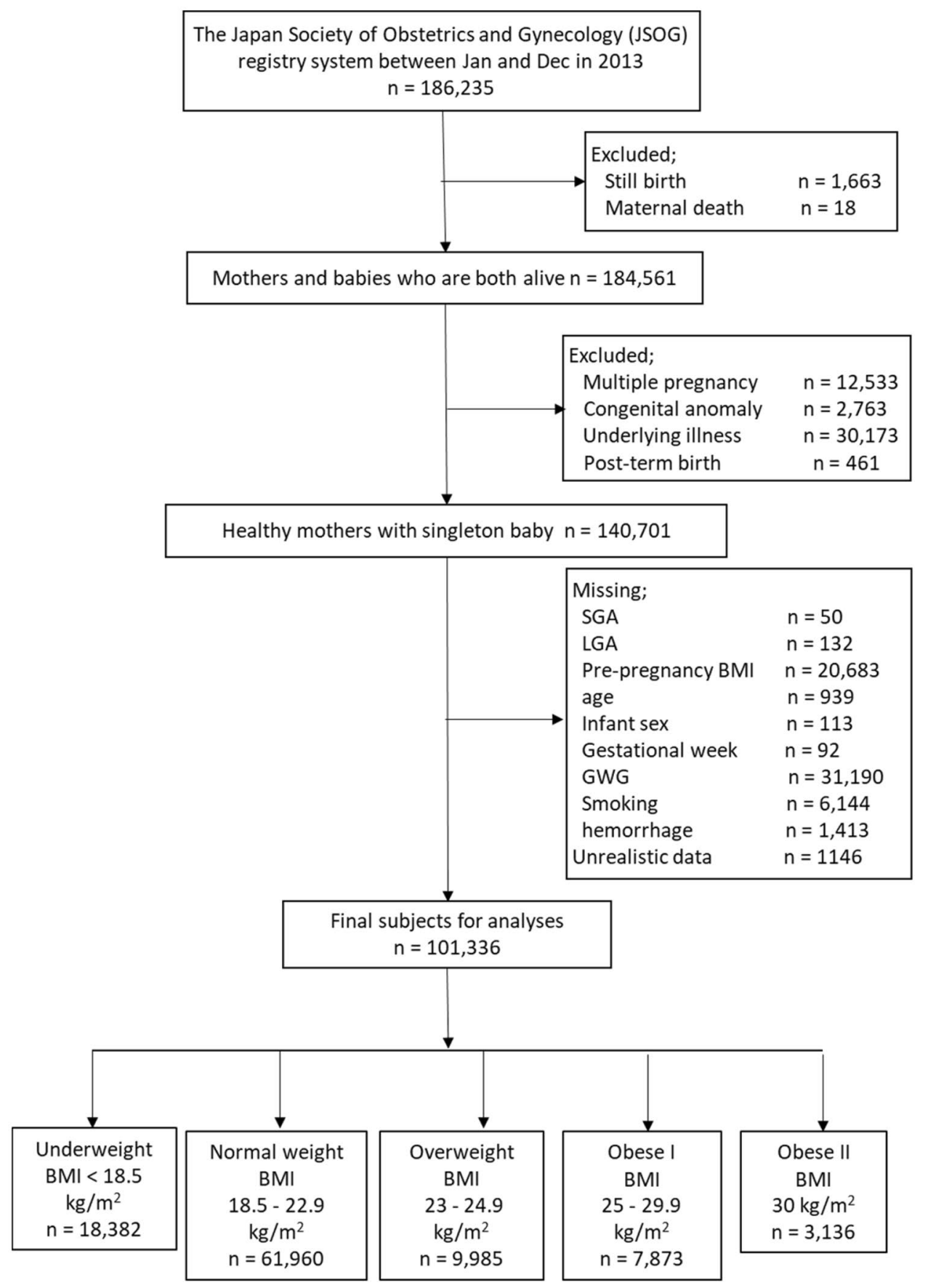

Figure 2. Flow chart of study enrollment.

as BMI $>25 \mathrm{~kg} / \mathrm{m}^{2}$ and that for overweight status as BMI $23-25 \mathrm{~kg} / \mathrm{m}^{2}$ for people from the Asia-Oceania region. In this study, based on the Asian-specific pre-pregnancy BMI classification recommended by the WHO, 18,382 women were underweight with BMI $<18.5 \mathrm{~kg} / \mathrm{m}^{2}, 61,960$ women were normal weight with BMI $18.5-22.9 \mathrm{~kg} / \mathrm{m}^{2}$, 9,985 women were overweight with BMI $23-24.9 \mathrm{~kg} / \mathrm{m}^{2}, 7,873$ women were obese with BMI $25-29.9 \mathrm{~kg} / \mathrm{m}^{2}$, and 3,136 women were obese with $\mathrm{BMI} \geq 30 \mathrm{~kg} / \mathrm{m}^{2}$.

Pregnancy outcomes. We determined pregnancy outcomes, including SGA $(<10$ th percentile of infant growth curve $)^{36}$, LGA ( $>90$ th percentile of infant growth curve $)^{36}$, LBW $(<2,500 \mathrm{~g})$, macrosomia (birth weight $>4,000 \mathrm{~g}$ ), preterm birth ( $<37$ gestational weeks), GDM (based on 75 -g oral glucose tolerance test and at least one of the following: $\geq 92 \mathrm{mg} / \mathrm{dL}$ fasting glucose, $\geq 180 \mathrm{mg} / \mathrm{dL}$ glucose at $1 \mathrm{~h}$, or $\geq 153 \mathrm{mg} / \mathrm{dL}$ glucose at $2 \mathrm{~h}$ ), pregnancy-induced hypertension (systolic blood pressure $\geq 140$ and/or diastolic blood pressure $\geq 90 \mathrm{mmHg}$ after 20 weeks of gestation); PPH-VD (blood loss $\geq 500 \mathrm{~mL}$ ), and PPH-CS (blood loss $\geq 1,000 \mathrm{~mL}$ ). 
Covariates. The items investigated in this study included maternal age, parity, gestational week at delivery, height, pre-pregnancy body weight, body weight at delivery, delivery mode, sex of infant, and birth weight. Gestational weeks were determined based on the last menstrual period.

Statistical analyses. All analyses conducted were stratified by Asian-specific BMI classification (i.e., $<18.5 \mathrm{~kg} / \mathrm{m}^{2}$ for underweight, $18.5-22.9 \mathrm{~kg} / \mathrm{m}^{2}$ for normal weight, $23-24.9 \mathrm{~kg} / \mathrm{m}^{2}$ for overweight, $25-29.9 \mathrm{~kg} / \mathrm{m}^{2}$ for obese I, $30<\mathrm{kg} / \mathrm{m}^{2}$ for obese II) ${ }^{22}$. To assess the applicability of the current Japanese recommendations for GWG, we estimated the risks of pregnancy outcomes associated with GWG below and above the range recommended in the Japanese guidelines compared to GWG within the recommended range in underweight (i.e., $9-12 \mathrm{~kg}$ ) and normal-weight women (i.e., $7-12 \mathrm{~kg}$ ). For overweight and obese women, the guidelines recommend weight gain of up to $5 \mathrm{~kg}$ depending on individual characteristics. Accordingly, we calculated odds ratios for various pregnancy outcomes below and above a weight gain of $5 \mathrm{~kg}$ in overweight and obese women. We used a logistic regression model to calculate ORs together with 95\% CIs within each BMI category, after adjusting for covariates. The covariates included maternal age, pre-pregnancy BMI, parity, delivery method, smoking, and gestational week, and were selected by each stepwise multivariable model. Among these, as maternal weight is a risk factor for the outcomes of interest, continuous pre-pregnancy BMI as well as GWG classification were included in stepwise models. For the outcome of preterm birth, as it was defined based on gestational week $<37$ and was strongly correlated with gestational week, gestational week was excluded from the covariates in the selection models. Similarly, as the outcomes PPH-VD and PPH-CS were strongly explained by delivery method, these variables were also excluded from the covariates in the selection models.

To estimate the risk of any adverse pregnancy outcome based on pre-pregnancy BMI levels, crude ROC curves and adjusted ROC curves were drawn adjusting for covariates selected in stepwise multivariable logistic regression models to investigate the risks of various pregnancy outcomes associated with the continuous GWG variable. The covariate-adjusted ROC curves and AUC were calculated based on the method of Pepe et al. ${ }^{37}$. The AUC is considered an effective measure of accuracy with a meaningful interpretation, and AUC values of 0.6-0.8 are considered acceptable for prediction of GWG based on Hosmer and Lemeshow ${ }^{38}$. In determining the optimal GWG range, we calculated Youden's index as sensitivity + specificity - 1 based on the reliable sensitivity and specificity estimated using the bootstrap method ${ }^{39}$. Youden's index indicates the maximum vertical distance of the ROC curve from a point $(\mathrm{x}, \mathrm{y})$ on the diagonal line, and thus maximizes the difference between the true positive fraction and false positive fraction ${ }^{23}$. We calculated the adjusted cutoff points of GWG corresponding to the largest Youden's index value and determined the optimal GWG for a pregnancy outcome with its lower boundary of the $95 \%$ CI of AUC $>0.6$. We performed sensitivity analyses both including and excluding women with preterm birth to estimate optimal GWG ranges.

All data were analyzed using SAS version 9.4 for Windows (SAS Institute, Cary, NC) and Stata version 14.2 (Stata Corp., College Station, TX). In all analyses, $P<0.05$ was taken to indicate statistical significance.

Received: 29 July 2019; Accepted: 6 November 2019;

Published online: 21 November 2019

\section{References}

1. The Ministry of Health Labour and Welfare. Nutrition Survey, http://www.mhlw.go.jp/bunya/kenkou/kenkou_eiyou_chousa.html (2017).

2. Enomoto, K. et al. Pregnancy Outcomes Based on Pre-Pregnancy Body Mass Index in Japanese Women. PLoS One. 11, 10.0151371/ journal.pone.0157081 (2016).

3. Barker, D. J. The origins of the developmental origins theory. J Intern Med. 261, 412-417 (2007).

4. Gillman, M. W. et al. Meeting report on the 3rd International Congress on Developmental Origins of Health and Disease (DOHaD). Pediatr Res. 61, 625-629 (2007).

5. OECD home. OECD Family Database. CO1.3: Low birth weight, http://www.oecd.org/els/family/CO_1_3_Low_birth_weight.pdf (2018).

6. Nomura, K. et al. For making a declaration of countermeasures against the falling birth rate from the Japanese Society for Hygiene: summary of discussion in the working group on academic research strategy against an aging society with low birth rate. Environ Health Prev Med. 24, 14, https://doi.org/10.1186/s12199-019-0768-x (2019).

7. Goldstein, R. F. et al. Association of Gestational Weight Gain With Maternal and Infant Outcomes: A Systematic Review and Metaanalysis. JAMA. 317, 2207-2225, https://doi.org/10.1001/jama.2017.3635 (2017).

8. The Ministry of Health Labour and Welfare. "Healthy Families (Sukoyaka. Oyako) 21", http://sukoyaka21.jp (2015).

9. Promotion Committee of the "Healthy Families (Sukoyaka. Oyako) 21" Project, http://rhino.med.yamanashi.ac.jp/sukoyaka/ninpu_ syoku.html (2006).

10. Ogawa, K., Morisaki, N., Sago, H., Fujiwara, T. \& Horikawa, R. Association between women's perceived ideal gestational weight gain during pregnancy and pregnancy outcomes. Sci Rep. 8, 11574, https://doi.org/10.1038/s41598-018-29936-z (2018).

11. Nohr, E. A. et al. Combined associations of prepregnancy body mass index and gestational weight gain with the outcome of pregnancy. Am J Clin Nutr. 87, 1750-1759 (2008).

12. Huang, X. et al. Gestational weight gain in Chinese women-results from a retrospective cohort in Changsha, China. BMC Pregnancy Childbirth. 18, 185, https://doi.org/10.1186/s12884-018-1833-y (2018).

13. Oken, E., Kleinman, K. P., Belfort, M. B., Hammitt, J. K. \& Gillman, M. W. Associations of gestational weight gain with short- and longer-term maternal and child health outcomes. Am J Epidemiol. 170, 173-180 (2009).

14. Fujiwara, K. et al. Associations of maternal pre-pregnancy underweight with small-for-gestational-age and spontaneous preterm birth, and optimal gestational weight gain in Japanese women. J Obstet Gynaecol Res. 40, 988-994 (2014).

15. Wataba, K. et al. Impact of prepregnant body mass index and maternal weight gain on the risk of pregnancy complications in Japanese women. Acta Obstet Gynecol Scand. 85, 269-276 (2006).

16. Tan, J. et al. The pattern of gestational weight gains among Chinese women: a repeated measure analysis. Sci Rep. 8, 15865, https:// doi.org/10.1038/s41598-018-34227-8 (2018)

17. Wie, J. H. et al. Is it appropriate for Korean women to adopt the 2009 Institute of Medicine recommendations for gestational weight gain? PLoS One. 12; https://doi.org/10.1371/journal.pone.0181164 (2017)

18. Institute of Medicine. Weight Gain During Pregnancy: Reexamining the Guidelines. (National Academies Press, 2009). 
19. Ren, M. et al. Excessive gestational weight gain in accordance with the IOM criteria and the risk of hypertensive disorders of pregnancy: a meta-analysis. BMC Pregnancy Childbirth. 18, 281 (2018).

20. Suzuki, S. Optimal Weight Gain During Pregnancy in Japanese Women. J Clin Med Res. 8, 787-792 (2016).

21. Nomura, K. et al. Investigation of optimal weight gain during pregnancy for Japanese Women. Sci Rep. 7, 2569, https://doi. org/10.1038/s41598-017-02863-1 (2017).

22. International Obesity Taskforce. World Health Organization Western Pacific region. The Asia-Pacific perspective: Redefining obesity and its treatment (2000).

23. Hajian-Tilaki, K. Receiver Operating Characteristic (ROC) Curve Analysis for Medical Diagnostic Test Evaluation. Caspian J Intern Med. 4, 627-35 (2013).

24. Morisaki, N. et al. Pre-pregnancy BMI-specific optimal gestational weight gain for women in Japan. J Epidemiol. 27, 492-498 (2017).

25. Hirooka-Nakama, J. et al. Optimal weight gain in obese and overweight pregnant Japanese women. Endocr J. 65, 557-567 (2018).

26. Ee, T. X. et al. Determining optimal gestational weight gain in a multiethnic Asian population. J Obstet Gynaecol Res. 40, 1002-1008 (2014).

27. Choi, S. K. et al. Determining optimal gestational weight gain in the Korean population: a retrospective cohort study. Reprod Biol Endocrinol. 15, 67 (2017).

28. Vital statistics Ministry of Health, Labour, and Welfare, https://www.mhlw.go.jp/toukei/list/81-1a.html (2019).

29. Toma, R., Aoki, S., Fujiwara, K. \& Hirahara, F. Associations of pre-pregnancy obesity with adverse pregnancy outcomes and the optimal gestational weight gain in Japanese women. Clin Exp Obstet Gynecol. 44, 190-194 (2017).

30. Suzuki, S., Shin, S. \& Araki, T. Weight control during pregnancy (in Japanese) Obstet Gynecol Pract (Tokyo) 45, 927-932 (1996).

31. Li, C., Liu, Y. \& Zhang, W. Joint and Independent Associations of Gestational Weight Gain and Pre-Pregnancy Body Mass Index with Outcomes of Pregnancy in Chinese Women: A Retrospective Cohort Study. PLoS One. 10, https://doi.org/10.1371/journal. pone.0136850 (2015).

32. Gujral, U. P. et al. Ethnic differences in the prevalence of diabetes in underweight and normal weight individuals: The CARRS and NHANES studies. Diabetes Res Clin Pract. 146, 34-40, https://doi.org/10.1016/j.diabres.2018.09.011 (2018).

33. Suzuki, K., Shinohara, R., Sato, M., Otawa, S. \& Yamagata, Z. Association Between Maternal Smoking During Pregnancy and Birth Weight: An Appropriately Adjusted Model From the Japan Environment and Children's Study. J Epidemiol. 26, 371-377, https://doi. org/10.2188/jea.JE20150185 (2016)

34. Morisaki, N. et al. Optimal protein intake during pregnancy for reducing the risk of fetal growth restriction: the Japan Environment and Children's Study. Br J Nutr. 120, 1432-1440, https://doi.org/10.1017/S000711451800291X (2018).

35. Rasmussen, K. M. \& Yaktine, A. L. Weight gain during pregnancy: reexamining the guidelines. Washington DC: National Academy of Sciences (2009).

36. Itabashi, K. et al. Introduction of new Birth Size Standards by Gestational Age for Japanese Neonates[atarashii zaitaikikannbetsushusseijitaikakuhyoujunnchi no dounyuu nitsuite]. J Jpn Pediatr Soc. 114, 1271-1293 (2010).

37. Pepe, M., Longton, G. \& Janes, H. Estimation and comparison of receiver operating characteristic curves. The Stata Journal 1-16 (2009).

38. Hosmer, D. W. \& Lemeshow, S. Assessing the Fit of the Model. Applied Logistic regression. John Wiley\&Sons, 160-164 (2000).

39. Youden, W. J. Index for rating diagnostic tests. Cancer. 3, 32-35 (1950).

\section{Acknowledgements}

This study was funded by the Japanese Ministry of Education, Science, Sports and Culture, Grant in Scientific Research (B), Number 16H0526. We thank Dr. Toshiya Itoh who belongs to department of OBGY, Hamamatsu University School of Medicine, for his assistance to obtain data collection.

\section{Author contributions}

K. Nomura conceived the study design which was advised by S.S. and H.I.; H.I. obtained the data; K. Nagashima wrote statistical program and K.Nomura analyzed the data; K. Nomura edited the draft and had primary responsibility for the final content. K. Nagahima reanalyzed data and confirmed that all the results were valid; S.S. and H.I. critically reviewed the draft and provided important intellectual content to the interpretation of the results. All the authors read and approved the final manuscript.

\section{Competing interests}

The authors declare no competing interests.

\section{Additional information}

Correspondence and requests for materials should be addressed to K.N.

Reprints and permissions information is available at www.nature.com/reprints.

Publisher's note Springer Nature remains neutral with regard to jurisdictional claims in published maps and institutional affiliations.

Open Access This article is licensed under a Creative Commons Attribution 4.0 International License, which permits use, sharing, adaptation, distribution and reproduction in any medium or format, as long as you give appropriate credit to the original author(s) and the source, provide a link to the Creative Commons license, and indicate if changes were made. The images or other third party material in this article are included in the article's Creative Commons license, unless indicated otherwise in a credit line to the material. If material is not included in the article's Creative Commons license and your intended use is not permitted by statutory regulation or exceeds the permitted use, you will need to obtain permission directly from the copyright holder. To view a copy of this license, visit http://creativecommons.org/licenses/by/4.0/.

(C) The Author(s) 2019 